\title{
ITERATIVE ALGORITHM FOR SOLVING MIXED QUASI-VARIATIONAL-LIKE INEQUALITIES WITH SKEW-SYMMETRIC TERMS IN BANACH SPACES
}

\author{
LU-CHUAN CENG, QAMRUL HASAN ANSARI, AND JEN-CHIH YAO
}

Received 1 April 2006; Accepted 28 May 2006

We develop an iterative algorithm for computing the approximate solutions of mixed quasi-variational-like inequality problems with skew-symmetric terms in the setting of reflexive Banach spaces. We use Fan-KKM lemma and concept of $\eta$-cocoercivity of a composition mapping to prove the existence and convergence of approximate solutions to the exact solution of mixed quasi-variational-like inequalities with skew-symmetric terms. Furthermore, we derive the posteriori error estimates for approximate solutions under quite mild conditions.

Copyright (c) 2006 Lu-Chuan Ceng et al. This is an open access article distributed under the Creative Commons Attribution License, which permits unrestricted use, distribution, and reproduction in any medium, provided the original work is properly cited.

\section{Introduction}

It is well known that variational inequality theory, as a very effective and powerful tool, not only has stimulated new results dealing with partial differential equations, but also has been applied to a large variety of problems arising in mechanics, contact problems in elasticity, optimization and control problems, management science, operations research, general equilibrium problems in economics and transportation, unilateral problems, obstacle problems, and so forth. Because of its wide applications, the classical variational inequality has been studied and generalized in various directions previously by many authors. Among these generalizations, mixed variational-like inequality problem is of interest and importance. One of the most important and interesting problems in the theory of variational inequality is the development of an efficient and implementable algorithm for solving variational inequality and its generalizations. These methods include projection method and its variant forms, linear approximation, descent and Newton's methods, and method based on the auxiliary principle technique. The method based on auxiliary principle technique was first suggested by Glowinski et al. [6] for solving variational inequalities in 1981. Subsequently, it has been used to solve a number of generalizations

Hindawi Publishing Corporation Journal of Inequalities and Applications Volume 2006, Article ID 82695, Pages 1-16 DOI 10.1155/JIA/2006/82695 
of classical variational inequalities; see, for example, $[1,4,8,14-16]$ and the references therein.

Let $H$ be a real Hilbert space whose inner product and norm are denoted by $\langle\cdot, \cdot\rangle$, and $\|\cdot\|$, respectively. Let $D$ be a nonempty convex subset of $H$. Let $T, A: D \rightarrow H$, and $\eta: D \times D \rightarrow H$ be mappings, and let $f: D \rightarrow \mathbb{R}$ be a real-valued function. Recently, Ansari and Yao [1] (see also [10]) considered and studied the mixed variational-like inequality problem (MVLIP), which is to find $u^{*} \in D$ such that

$$
\left\langle T u^{*}-A u^{*}, \eta\left(v, u^{*}\right)\right\rangle+f(v)-f\left(u^{*}\right) \geq 0, \quad \forall v \in D .
$$

When $A \equiv 0$, this problem reduces to the following problem considered by Dien [3], Noor [11] and Siddiqi et al. [12]: find $u^{*} \in D$ such that

$$
\left\langle T u^{*}, \eta\left(v, u^{*}\right)\right\rangle+f(v)-f\left(u^{*}\right) \geq 0, \quad \forall v \in D .
$$

In [1], Ansari and Yao introduced the concepts of $\eta$-cocoercivity, $\eta$-strong monotonicity, and $\eta$-strong convexity of a mapping, which generalize the definitions of cocoercivity $[13,17]$, strong monotonicity [9], and strong convexity [9], respectively. It is easy to see that $\eta$-cocoercivity is an intermediate concept that lies between $\eta$-strong monotonicity and $\eta$-monotonicity. They applied the auxiliary variational inequality technique to suggest an iterative algorithm for finding the approximate solutions of MVLIP and proved that these approximate solutions converge to the exact solution of MVLIP.

Motivated and inspired by the work in [1], we consider and study a class of mixed quasi-variational-like inequality problems in the setting of Banach spaces.

Let $D$ be a nonempty convex subset of a real Banach space $B$, let $B^{*}$ be the topological dual space of $B$, and let $\langle u, v\rangle$ be the duality pairing between $u \in B^{*}$ and $v \in B$. Let $T$, $A: D \rightarrow B^{*}, N: B^{*} \times B^{*} \rightarrow B^{*}$, and $\eta: D \times D \rightarrow B$ be mappings and $w^{*} \in B^{*}$. Let $\varphi: B \times$ $B \rightarrow \mathbb{R} \cup\{+\infty\}$ be a real bifunction. The mixed quasi-variational-like inequality problem (MQVLIP) is to find $u \in D$ such that

$$
\left\langle N(T u, A u)-w^{*}, \eta(v, u)\right\rangle+\varphi(v, u)-\varphi(u, u) \geq 0, \quad \forall v \in D .
$$

This problem was first introduced and studied by Ding and Yao [4]. They applied the auxiliary variational inequality technique to suggest an iterative algorithm for computing the approximate solutions of MQVLIP (1.3), and provided the convergence criteria of approximate solutions to the exact solution of MQVLIP (1.3).

In this paper, we propose a new iterative algorithm for computing the approximate solutions of MQVLIP (1.3) with skew-symmetric term $\varphi(\cdot, \cdot)$ in the setting of a reflexive Banach space $B$. Our proposed iterative algorithm can be seen as an extension and generalization of Ansari and Yao's [1] iterative algorithm. We employ Fan-KKM lemma and concept of $\eta$-cocoercivity of a composition mapping to prove the existence and convergence of approximate solutions to the exact solution of MQVLIP (1.3) with skewsymmetric term $\varphi(\cdot, \cdot)$. Compared with Ansari and Yao [1, Theorem 3.2], our results improve and generalize their Theorem 3.2 in the following aspects: (i) the MQVLIP (1.3) is more general than MVLIP considered by them; (ii) we remove boundedness assumption on $D$; (iii) our convergence criteria for approximate solutions are very different from 
their ones because of the appearance of skew-symmetric term $\varphi(\cdot, \cdot)$ in MQVLIP (1.3); (iv) the technique of our proof is very different from the one of their proof since reflexive Banach space is more general than Hilbert space; (v) we provide the posteriori error estimates for approximate solutions under quite mild conditions. Hence the results of this paper also extend and generalize the results of Zhu and Marcotte [17].

\section{Preliminaries}

In this section we will recall the following definitions and some known results.

Definition 2.1. Let $D$ be a nonempty subset of a Banach space $B$ with the dual space $B^{*}$. Let $T: D \rightarrow B^{*}$ and $\eta: D \times D \rightarrow B$ be two mappings. Then,

(i) $T$ is called $\eta$-monotone, if

$$
\langle T u-T v, \eta(u, v)\rangle \geq 0, \quad \forall u, v \in D
$$

(ii) $T$ is called $\eta$-strongly monotone, if there exists a constant $\beta>0$, such that

$$
\langle T u-T v, \eta(u, v)\rangle \geq \beta\|u-v\|^{2}, \quad \forall u, v \in D ;
$$

(iii) $T$ is called $\eta$-cocoercive, if there exists a constant $\xi>0$, such that

$$
\langle T u-T v, \eta(u, v)\rangle \geq \xi\|T u-T v\|^{2}, \quad \forall u, v \in D ;
$$

(iv) $T$ is called $\eta$-relaxed monotone, if there exists a constant $\xi>0$, such that

$$
\langle T u-T v, \eta(u, v)\rangle \geq \beta\|u-v\|^{2}, \quad \forall u, v \in D ;
$$

(v) $T$ is called Lipschitz continuous, if there exists a constant $L>0$, such that

$$
\|T u-T v\| \leq L\|u-v\|, \quad \forall u, v \in D
$$

(vi) $\eta$ is called Lipschitz continuous, if there exists a constant $\delta>0$, such that

$$
\|\eta(u, v)\| \leq \delta\|u-v\|, \quad \forall u, v \in D .
$$

We illustrate hereafter the relationships among $\eta$-monotonicity, $\eta$-strong monotonicity, $\eta$-cocoercivity, and Lipschitz continuity as follows:

(i) $\eta$-strong monotonicity $\Longrightarrow \eta$-monotonicity $\Longleftarrow \eta$-cocoercivity;

(ii)

$$
\begin{aligned}
& T \text { is } \eta \text {-strong monotone } \\
& T \text { is Lipschitz continuous }
\end{aligned} \Longrightarrow T \text { is } \eta \text {-cocoercive; }
$$

(iii)

$T$ is $\eta$-cocoercive $\eta$ is Lipschitz continuous $\Longrightarrow T$ is Lipschitz continuous. 
4 Iterative algorithm for solving MQVLIP

Definition 2.2. The bifunction $\varphi: B \times B \rightarrow \mathbb{R} \bigcup\{+\infty\}$ is said to be skew-symmetric if

$$
\varphi(u, u)-\varphi(u, v)-\varphi(v, u)+\varphi(v, v) \geq 0, \quad \forall u, v \in B
$$

The skew-symmetric bifunctions have properties which can be considered analogs of monotonicity of gradient and nonnegativity of a second derivative for convex functions. For properties and applications of skew-symmetric bifunction, reader may consult [2].

Definition 2.3. Let $D$ be a convex subset of a Banach space $B$ and let $K: D \rightarrow \mathbb{R}$ be a Fréchet differentiable function. $K$ is said to be

(i) $\eta$-convex [7], if

$$
K(v)-K(u) \geq\left\langle K^{\prime}(u), \eta(v, u)\right\rangle, \quad \forall u, v \in D
$$

(ii) $\eta$-strongly convex [11], if there exists a constant $\mu>0$, such that

$$
K(v)-K(u)-\left\langle K^{\prime}(u), \eta(v, u)\right\rangle \geq \frac{\mu}{2}\|u-v\|^{2}, \quad \forall u, v \in D
$$

In particular, if $\eta(v, u)=v-u$ for all $v, u \in D$, then $K$ is said to be strongly convex.

Proposition 2.4. Let $K$ be a differentiable $\eta$-strongly convex functional with constant $\mu>0$ on a convex subset $D$ of $B$, and let $\eta: D \times D \rightarrow B$ be a mapping such that $\eta(u, v)+\eta(v, u)=0$, for all $u, v \in D$. Then $K^{\prime}$ is $\eta$-strongly monotone with constant $\mu>0$.

Proof. Since $K$ is $\eta$-strongly convex, we deduce that for each $u, v \in D$

$$
\begin{aligned}
& K(v)-K(u)-\left\langle K^{\prime}(u), \eta(v, u)\right\rangle \geq \frac{\mu}{2}\|u-v\|^{2}, \\
& K(u)-K(v)-\left\langle K^{\prime}(v), \eta(u, v)\right\rangle \geq \frac{\mu}{2}\|v-u\|^{2} .
\end{aligned}
$$

Adding these two inequalities and using the condition that $\eta(u, v)+\eta(v, u)=0$, we obtain

$$
\left\langle K^{\prime}(v)-K^{\prime}(u), \eta(v, u)\right\rangle \geq \mu\|v-u\|^{2} .
$$

A function $F: D \rightarrow \mathbb{R}$ is called weakly sequentially continuous at $x_{0} \in D$, if $F\left(x_{k}\right) \rightarrow$ $F\left(x_{0}\right)(n \rightarrow \infty)$ for each sequence $\left\{x_{k}\right\} \subset D$ converging weakly to $x_{0} . F$ is called weakly sequentially continuous on $D$, if it is weakly sequentially continuous at each point of $D$.

Lemma 2.5. Let $\eta(v, \cdot): D \rightarrow B$ and $K^{\prime}$ be sequentially continuous from the weak topology to the weak topology and from the weak topology to the strong topology, respectively, where $v$ is any fixed point in $D$. Then the function $g: D \rightarrow \mathbb{R}$, defined as $g(u)=\left\langle K^{\prime}(u), \eta(v, u)\right\rangle$ for each fixed $v \in D$, is weakly sequentially continuous on $D$. 
Proof. Let $u$ be any given point in $D$, and let $\left\{u_{n}\right\} \subset D$ be any sequence converging weakly to $u$. Then, $\left\|K^{\prime}\left(u_{n}\right)-K^{\prime}(u)\right\| \rightarrow 0(n \rightarrow \infty)$, and $\left\{\eta\left(v, u_{n}\right)\right\}$ converges weakly to $\eta(v, u)$ for each fixed $v \in D$. Observe that

$$
\begin{aligned}
\left|g\left(u_{n}\right)-g(u)\right| & =\left|\left\langle K^{\prime}\left(u_{n}\right)-K^{\prime}(u), \eta\left(v, u_{n}\right)\right\rangle+\left\langle K^{\prime}(u), \eta\left(v, u_{n}\right)-\eta(v, u)\right\rangle\right| \\
& \leq\left\|K^{\prime}\left(u_{n}\right)-K^{\prime}(u)||\left|\eta\left(v, u_{n}\right) \|+\right|\left\langle K^{\prime}(u), \eta\left(v, u_{n}\right)-\eta(v, u)\right\rangle \mid\right. \\
& \longrightarrow 0 \text { as } n \longrightarrow \infty .
\end{aligned}
$$

Therefore, the conclusion immediately follows.

For each $D \subset B$, we denote by $\operatorname{co}(D)$ the convex hull of $D$. A point-to-set mapping $G: D \rightarrow 2^{B}$ is called a KKM mapping if, for every finite subset $\left\{u_{1}, u_{2}, \ldots, u_{k}\right\}$ of $D$,

$$
\operatorname{co}\left(\left\{u_{1}, u_{2}, \ldots, u_{k}\right\}\right) \subset \bigcup_{i=1}^{k} G\left(x_{i}\right) .
$$

In the next section, we will use the following result.

LEMMA 2.6 (Fan-KKM [5]). Let D be an arbitrary nonempty subset in a topological vector space $E$, and let $G: D \rightarrow 2^{E}$ be a KKM mapping. If $G(u)$ is closed for all $u \in D$ and is compact for at least one $u \in D$, then $\bigcap_{u \in D} G(u) \neq \varnothing$.

\section{Iterative algorithm and its convergence}

In this section, following the approach of Ansari and Yao [1], we will apply the auxiliary variational inequality technique to suggest a general algorithm for finding approximate solutions of MQVLIP (1.3) with skew-symmetric term $\varphi(\cdot, \cdot)$. Moreover, we will also study convergence analysis of proposed algorithm.

Algorithm 3.1. Let $K: D \rightarrow \mathbb{R}$ be a given Fréchet differentiable functional, let $\left\{\rho_{n}\right\}_{n=0}^{\infty}$ be a sequence of positive parameters, and let $u_{0}$ be any initial guess in $D$. For each given iterate $u_{n} \in D$, consider the following auxiliary variational inequality problem: find $u_{n+1} \in D$, such that

$$
\begin{gathered}
\left\langle\rho_{n} N\left(T u_{n}, A u_{n}\right)-\rho_{n} w^{*}+K^{\prime}\left(u_{n+1}\right)-K^{\prime}\left(u_{n}\right), \eta\left(v, u_{n+1}\right)\right\rangle \\
+\rho_{n} \varphi\left(v, u_{n+1}\right)-\rho_{n} \varphi\left(u_{n+1}, u_{n+1}\right) \geq 0, \quad \forall v \in D,
\end{gathered}
$$

where $K^{\prime}(u)$ is the Fréchet derivative of a function $K: D \rightarrow \mathbb{R}$ at $u \in D$.

Theorem 3.2. Let $D$ be a nonempty closed convex subset of a reflexive Banach space $B$ with the dual space $B^{*}$. Let $T, A: D \rightarrow B^{*}, N: B^{*} \times B^{*} \rightarrow B^{*}$, and $\eta: D \times D \rightarrow B$ be mappings. Let $w^{*} \in B^{*}$ and $\varphi: B \times B \rightarrow \mathbb{R} \bigcup\{+\infty\}$ be skew-symmetric and weakly continuous, such that for each $v \in B$, int $(\operatorname{dom} \varphi(\cdot, v)) \cap D \neq \varnothing$ and $\varphi(\cdot, v)$ is proper convex. Suppose that $K: D \rightarrow \mathbb{R}$ is $\eta$-strongly convex with constant $\mu>0$, and $K^{\prime}$ is sequentially continuous from 


\section{Iterative algorithm for solving MQVLIP}

the weak topology to the strong topology. Suppose also that

(i) the mapping $u \rightarrow N(T u, A u)$ is $\eta$-cocoercive with constant $v>0$;

(ii) $\eta$ is Lipschitz continuous with constant $\delta>0$, such that

(a) $\eta(u, v)=\eta(u, z)+\eta(z, v)$ for each $u, v, z \in D$,

(b) $\eta(\cdot, \cdot)$ is affine in the first variable,

(c) for each fixed $v \in D, u \rightarrow \eta(v, u)$ is sequentially continuous from the weak topology to the weak topology;

(iii) for each fixed $n \geq 0$ and $z \in D$,

$$
\left\{u \in D:\left\langle\rho_{n} N(T z, A z)-\rho_{n} w^{*}+K^{\prime}(u)-K^{\prime}(z), \eta(v, u)\right\rangle+\rho_{n} \varphi(v, u)-\rho_{n} \varphi(u, u) \geq 0\right\}
$$

is bounded for at least one $v \in D$.

Then, there is a unique solution $u_{n+1} \in D$ to problem (3.1) for each given iterate $u_{n}$. If

$$
\rho_{n+1} \leq \rho_{n}, \quad c_{1}<\rho_{n}<\frac{2 \mu}{\delta}\left(\frac{1}{v}+c_{2}\right), \quad n \geq 0, \text { for some } c_{1}, c_{2}>0
$$

then

(I) $\left\{u_{n}\right\}$ is bounded;

(II) $\lim _{n \rightarrow \infty}\left\|u_{n+1}-u_{n}\right\|=0$;

(III) $\left\{u_{n}\right\}$ converges weakly to a solution of MQVLIP (1.3) provided that the mapping $u \rightarrow$ $N(T u, A u)$ is sequentially continuous from the weak topology to the strong topology.

Proof. Existence of solutions of problem (3.1). For the sake of simplicity, we rewrite (3.1) as follows. Find $\bar{u} \in D$ such that

$$
\left\langle\rho_{n} N\left(T u_{n}, A u_{n}\right)-\rho_{n} w^{*}+K^{\prime}(\bar{u})-K^{\prime}\left(u_{n}\right), \eta(v, \bar{u})\right\rangle+\rho_{n} \varphi(v, \bar{u})-\rho_{n} \varphi(\bar{u}, \bar{u}) \geq 0, \quad \forall v \in D .
$$

For each fixed $n \geq 0$ and each $v \in D$, we define

$$
\begin{aligned}
G(v)=\{u \in D: & \left\langle\rho_{n} N\left(T u_{n}, A u_{n}\right)-\rho_{n} w^{*}+K^{\prime}(u)-K^{\prime}\left(u_{n}\right), \eta(v, u)\right\rangle \\
& \left.+\rho_{n} \varphi(v, u)-\rho_{n} \varphi(u, u) \geq 0\right\} .
\end{aligned}
$$

Note that, since $v \in G(v), G(v)$ is nonempty for each $v \in D$. Now, we claim that $G$ is a KKM mapping. Indeed, suppose that there exists a finite subset $\left\{v_{1}, v_{2}, \ldots, v_{k}\right\}$ of $D$ and that $\alpha_{i} \geq 0$, for all $i=1,2, \ldots, k$ with $\sum_{i=1}^{k} \alpha_{i}=1$ such that $\hat{u}=\sum_{i=1}^{k} \alpha_{i} v_{i} \notin G\left(v_{i}\right)$, for all $i=1,2, \ldots, k$, that is,

$$
\begin{array}{r}
\left\langle\rho_{n} N\left(T u_{n}, A u_{n}\right)-\rho_{n} w^{*}+K^{\prime}(\widehat{u})-K^{\prime}\left(u_{n}\right), \eta\left(v_{i}, \widehat{u}\right)\right\rangle \\
+\rho_{n} \varphi\left(v_{i}, \hat{u}\right)-\rho_{n} \varphi(\hat{u}, \widehat{u})<0, \quad \forall i=1,2, \ldots, k .
\end{array}
$$


Since $\varphi(\cdot, v)$ is proper convex for each $v \in B$, by virtue of assumptions (a) and (b) in (ii), we have

$$
\begin{aligned}
& 0=\left\langle\rho_{n} N\left(T u_{n}, A u_{n}\right)-\rho_{n} w^{*}+K^{\prime}(\hat{u})-K^{\prime}\left(u_{n}\right), \eta(\hat{u}, \hat{u})\right\rangle+\rho_{n} \varphi(\hat{u}, \hat{u})-\rho_{n} \varphi(\hat{u}, \hat{u}) \\
& =\left\langle\rho_{n} N\left(T u_{n}, A u_{n}\right)-\rho_{n} w^{*}+K^{\prime}(\widehat{u})-K^{\prime}\left(u_{n}\right), \eta\left(\sum_{i=1}^{k} \alpha_{i} v_{i}, \hat{u}\right)\right\rangle \\
& +\rho_{n} \varphi\left(\sum_{i=1}^{k} \alpha_{i} v_{i}, \hat{u}\right)-\rho_{n} \varphi(\hat{u}, \widehat{u}) \\
& \leq\left\langle\rho_{n} N\left(T u_{n}, A u_{n}\right)-\rho_{n} w^{*}+K^{\prime}(\widehat{u})-K^{\prime}\left(u_{n}\right), \sum_{i=1}^{k} \alpha_{i} \eta\left(v_{i}, \widehat{u}\right)\right\rangle \\
& +\rho_{n} \sum_{i=1}^{k} \alpha_{i} \varphi\left(v_{i}, \hat{u}\right)-\rho_{n} \varphi(\hat{u}, \hat{u}) \\
& =\sum_{i=1}^{k} \alpha_{i}\left[\left\langle\rho_{n} N\left(T u_{n}, A u_{n}\right)-\rho_{n} w^{*}+K^{\prime}(\widehat{u})-K^{\prime}\left(u_{n}\right), \eta\left(v_{i}, \widehat{u}\right)\right\rangle+\rho_{n} \varphi\left(v_{i}, \widehat{u}\right)-\rho_{n} \varphi(\hat{u}, \widehat{u})\right]<0,
\end{aligned}
$$

which yields a contradiction. Therefore, $G$ is a KKM mapping.

Since $K^{\prime}$ is sequentially continuous from the weak topology to the strong topology, and $\varphi(\cdot, \cdot)$ is weakly continuous on $B \times B$, so it follows from condition (ii)(c) and Lemma 2.5 that $G(v)$ is a weakly closed subset of $D$ for each $v \in D$. Moreover, from condition (iii) we know that $G(v)$ is weakly compact for at least one point $v \in D$. Hence, by using Lemma 2.6 we have $\bigcap_{v \in D} G(v) \neq \varnothing$, which clearly implies that there exists at least one solution to problem (3.1).

Uniqueness of solutions of problem (3.1). Let $\bar{u}$ and $\tilde{u}$ be two solutions of problem (3.1). Then,

$$
\begin{aligned}
& \left\langle\rho_{n} N\left(T u_{n}, A u_{n}\right)-\rho_{n} w^{*}+K^{\prime}(\bar{u})-K^{\prime}\left(u_{n}\right), \eta(v, \bar{u})\right\rangle+\rho_{n} \varphi(v, \bar{u})-\rho_{n} \varphi(\bar{u}, \bar{u}) \geq 0, \\
& \left\langle\rho_{n} N\left(T u_{n}, A u_{n}\right)-\rho_{n} w^{*}+K^{\prime}(\tilde{u})-K^{\prime}\left(u_{n}\right), \eta(v, \tilde{u})\right\rangle+\rho_{n} \varphi(v, \tilde{u})-\rho_{n} \varphi(\tilde{u}, \tilde{u}) \geq 0,
\end{aligned}
$$

for all $v \in D$.

Note that $\eta(u, v)=\eta(u, z)+\eta(z, v)$ for all $u, v, z \in D$ implies $\eta(u, v)=-\eta(v, u)$ for all $u, v \in D$ and that $\varphi(\cdot, \cdot)$ is skew-symmetric. Taking $v=\tilde{u}$ in (3.8) and $v=\bar{u}$ in (3.9), and adding these inequalities, we get

$$
\begin{aligned}
-\left\langle K^{\prime}(\bar{u})-K^{\prime}(\tilde{u}), \eta(\bar{u}, \tilde{u})\right\rangle= & \left\langle K^{\prime}(\bar{u}), \eta(\tilde{u}, \bar{u})\right\rangle+\left\langle K^{\prime}(\tilde{u}), \eta(\bar{u}, \tilde{u})\right\rangle \\
= & \left\langle\rho_{n} N\left(T u_{n}, A u_{n}\right)-\rho_{n} w^{*}+K^{\prime}(\bar{u})-K^{\prime}\left(u_{n}\right), \eta(\tilde{u}, \bar{u})\right\rangle \\
& +\left\langle\rho_{n} N\left(T u_{n}, A u_{n}\right)-\rho_{n} w^{*}+K^{\prime}(\tilde{u})-K^{\prime}\left(u_{n}\right), \eta(\bar{u}, \tilde{u})\right\rangle \\
\geq & \rho_{n}[\varphi(\bar{u}, \bar{u})-\varphi(\tilde{u}, \bar{u})-\varphi(\bar{u}, \tilde{u})+\varphi(\tilde{u}, \tilde{u})] \geq 0,
\end{aligned}
$$


8 Iterative algorithm for solving MQVLIP

which hence implies that

$$
\left\langle K^{\prime}(\bar{u})-K^{\prime}(\tilde{u}), \eta(\bar{u}, \tilde{u})\right\rangle \leq 0 .
$$

Since $K^{\prime}$ is $\eta$-strongly monotone with constant $\mu>0$ by Proposition 2.4, from (3.11) we obtain

$$
\mu\|\bar{u}-\tilde{u}\|^{2} \leq\left\langle K^{\prime}(\bar{u})-K^{\prime}(\tilde{u}), \eta(\bar{u}, \tilde{u})\right\rangle \leq 0,
$$

and so $\bar{u}=\tilde{u}$. This shows that the solution of problem (3.1) is unique.

Let $u^{*} \in D$ be any fixed solution of MQVLIP (1.3). Since $u_{n+1}$ is the unique solution to problem (3.1), we get

$$
\begin{gathered}
\left\langle K^{\prime}\left(u_{n+1}\right)-K^{\prime}\left(u_{n}\right), \eta\left(v, u_{n+1}\right)\right\rangle+\rho_{n}\left\langle N\left(T u_{n}, A u_{n}\right)-w^{*}, \eta\left(v, u_{n+1}\right)\right\rangle \\
+\rho_{n} \varphi\left(v, u_{n+1}\right)-\rho_{n} \varphi\left(u_{n+1}, u_{n+1}\right) \geq 0, \quad \forall v \in D .
\end{gathered}
$$

We consider a function $\Lambda$ defined by

$$
\Lambda(u, \rho)=\Phi(u)+\Omega(u, \rho)
$$

where $\Omega(u, \rho)=\rho\left[\left\langle N\left(T u^{*}, A u^{*}\right)-w^{*}, \eta\left(u, u^{*}\right)\right\rangle+\varphi\left(u, u^{*}\right)-\varphi\left(u^{*}, u^{*}\right)\right]$ and

$$
\Phi(u)=K\left(u^{*}\right)-K(u)-\left\langle K^{\prime}(u), \eta\left(u^{*}, u\right)\right\rangle \text {. }
$$

From $\eta$-strong convexity of $K$, we obtain

$$
\Phi\left(u_{n}\right) \geq\left(\frac{\mu}{2}\right)\left\|u_{n}-u^{*}\right\|^{2} \geq 0
$$

Since $\Omega\left(u_{n}, \rho_{n}\right)$ is nonnegative, we have

$$
\Lambda\left(u_{n}, \rho_{n}\right) \geq\left(\frac{\mu}{2}\right)\left\|u_{n}-u^{*}\right\|^{2} \geq 0
$$

Let us study the variation of $\Lambda$ for one stage of Algorithm 3.1:

$$
\Gamma_{n}^{n+1}=\Lambda\left(u_{n+1}, \rho_{n+1}\right)-\Lambda\left(u_{n}, \rho_{n}\right)
$$

Then we have $\Gamma_{n}^{n+1}=s_{1}+s_{2}+s_{3}$, where

$$
\begin{gathered}
s_{1}=K\left(u_{n}\right)-K\left(u_{n+1}\right)-\left\langle K^{\prime}\left(u_{n}\right), \eta\left(u_{n}, u_{n+1}\right)\right\rangle, \\
s_{2}=\left\langle K^{\prime}\left(u_{n}\right)-K^{\prime}\left(u_{n+1}\right), \eta\left(u^{*}, u_{n+1}\right)\right\rangle, \\
s_{3}=\rho_{n+1}\left[\left\langle N\left(T u^{*}, A u^{*}\right)-w^{*}, \eta\left(u_{n+1}, u^{*}\right)\right\rangle+\varphi\left(u_{n+1}, u^{*}\right)-\varphi\left(u^{*}, u^{*}\right)\right] \\
-\rho_{n}\left[\left\langle N\left(T u^{*}, A u^{*}\right)-w^{*}, \eta\left(u_{n}, u^{*}\right)\right\rangle+\varphi\left(u_{n}, u^{*}\right)-\varphi\left(u^{*}, u^{*}\right)\right] .
\end{gathered}
$$


By using $\eta$-strong convexity of $K$, we have $s_{1} \leq-(\mu / 2)\left\|u_{n+1}-u_{n}\right\|^{2}$. Also, since $\rho_{n+1} \leq \rho_{n}$ and $u^{*}$ is a solution of MQVLIP (1.3), we immediately derive

$$
s_{3} \leq \rho_{n}\left[\left\langle N\left(T u^{*}, A u^{*}\right)-w^{*}, \eta\left(u_{n+1}, u^{*}\right)\right\rangle+\varphi\left(u_{n+1}, u^{*}\right)-\varphi\left(u^{*}, u^{*}\right)\right] .
$$

Now, putting $u=u^{*}$ in (3.13), we get

$$
s_{2} \leq \rho_{n}\left[\left\langle N\left(T u_{n}, A u_{n}\right)-w^{*}, \eta\left(u^{*}, u_{n+1}\right)\right\rangle+\varphi\left(u^{*}, u_{n+1}\right)-\varphi\left(u_{n+1}, u_{n+1}\right)\right] .
$$

Thus, in terms of skew-symmetry of $\varphi$ we deduce from (3.20) and (3.21) that

$$
\begin{aligned}
s_{2}+s_{3} \leq & \rho_{n}\left[\left\langle N\left(T u^{*}, A u^{*}\right)-w^{*}, \eta\left(u_{n+1}, u^{*}\right)\right\rangle+\varphi\left(u_{n+1}, u^{*}\right)-\varphi\left(u^{*}, u^{*}\right)\right] \\
& +\rho_{n}\left[\left\langle N\left(T u_{n}, A u_{n}\right)-w^{*}, \eta\left(u^{*}, u_{n+1}\right)\right\rangle+\varphi\left(u^{*}, u_{n+1}\right)-\varphi\left(u_{n+1}, u_{n+1}\right)\right] \\
= & \rho_{n}\left[\left\langle N\left(T u^{*}, A u^{*}\right)-w^{*}, \eta\left(u_{n+1}, u^{*}\right)\right\rangle+\left\langle N\left(T u_{n}, A u_{n}\right)-w^{*}, \eta\left(u^{*}, u_{n+1}\right)\right\rangle\right] \\
& -\rho_{n}\left[\varphi\left(u^{*}, u^{*}\right)-\varphi\left(u_{n+1}, u^{*}\right)-\varphi\left(u^{*}, u_{n+1}\right)+\varphi\left(u_{n+1}, u_{n+1}\right)\right] \\
\leq & -\rho_{n}\left\langle N\left(T u^{*}, A u^{*}\right)-N\left(T u_{n}, A u_{n}\right), \eta\left(u^{*}, u_{n+1}\right)\right\rangle \\
= & -\rho_{n}\left\langle N\left(T u^{*}, A u^{*}\right)-N\left(T u_{n}, A u_{n}\right), \eta\left(u^{*}, u_{n}\right)\right\rangle \\
& -\rho_{n}\left\langle N\left(T u^{*}, A u^{*}\right)-N\left(T u_{n}, A u_{n}\right), \eta\left(u_{n}, u_{n+1}\right)\right\rangle \\
\leq & -\rho_{n} v\left\|N\left(T u^{*}, A u^{*}\right)-N\left(T u_{n}, A u_{n}\right)\right\|^{2} \quad(\text { using } \eta \text {-cocoercivity) } \\
& +\rho_{n} \delta\left\|N\left(T u^{*}, A u^{*}\right)-N\left(T u_{n}, A u_{n}\right)\right\|\left\|u_{n+1}-u_{n}\right\| \quad \text { (using Lipschitz continuity), }
\end{aligned}
$$

which hence implies that

$$
\begin{aligned}
\Gamma_{n}^{n+1}= & s_{1}+s_{2}+s_{3} \\
\leq & -\frac{\mu}{2}\left\|u_{n+1}-u_{n}\right\|^{2}-v \rho_{n}\left\|N\left(T u_{n}, A u_{n}\right)-N\left(T u^{*}, A u^{*}\right)\right\|^{2} \\
& +\delta \rho_{n}\left\|N\left(T u_{n}, A u_{n}\right)-N\left(T u^{*}, A u^{*}\right)\right\|\left\|u_{n+1}-u_{n}\right\| .
\end{aligned}
$$

Then, by using the inequality

$$
\begin{aligned}
\rho_{n} \| N & \left(T u_{n}, A u_{n}\right)-N\left(T u^{*}, A u^{*}\right)\|\| u_{n+1}-u_{n} \| \\
& \leq\left(\frac{\rho_{n}^{2}}{2} \omega\right)\left\|N\left(T u_{n}, A u_{n}\right)-N\left(T u^{*}, A u^{*}\right)\right\|^{2}+\left(\frac{\omega}{2}\right)\left\|u_{n+1}-u_{n}\right\|^{2},
\end{aligned}
$$


where $\omega$ is a positive number chosen so that $\omega<\mu / \delta$, we get

$$
\Gamma_{n}^{n+1} \leq-\left(\frac{\mu}{2}-\frac{\delta \omega}{2}\right)\left\|u_{n+1}-u_{n}\right\|^{2}-\rho_{n}\left(v-\frac{\delta \rho_{n}}{2 \omega}\right)\left\|N\left(T u_{n}, A u_{n}\right)-N\left(T u^{*}, A u^{*}\right)\right\|^{2}
$$

For $c_{1}<\rho_{n}<2 \omega / \delta\left(1 / v+c_{2}\right)$, where $c_{1}>0$ and $c_{2}>0$, we have

$$
\rho_{n}\left(v-\frac{\delta \rho_{n}}{2 \omega}\right)>c_{1}\left(v-\frac{\delta}{2 \omega} \cdot \frac{2 \omega}{\delta\left(1 / v+c_{2}\right)}\right)=\frac{c_{1} c_{2} v}{1 / v+c_{2}}
$$

and hence

$$
\Gamma_{n}^{n+1} \leq-\left(\frac{\mu}{2}-\frac{\delta \omega}{2}\right)\left\|u_{n+1}-u_{n}\right\|^{2}-\left(\frac{c_{1} c_{2} v}{\left(1 / v+c_{2}\right)}\right)\left\|N\left(T u_{n}, A u_{n}\right)-N\left(T u^{*}, A u^{*}\right)\right\|^{2}
$$

Thus, for $\omega<\mu / \delta, \Gamma_{n}^{n+1}$ is negative unless $u_{n+1}=u_{n}$ and $N\left(T u_{n}, A u_{n}\right)=N\left(T u^{*}, A u^{*}\right)$. Then, according to (3.13), $u_{n}$ is a solution of MQVLIP (1.3).

Note that the sequence $\left\{\Lambda\left(u_{n}, \rho_{n}\right)\right\}$ is strictly decreasing. But since it is positive, it must converge and the difference between two consecutive terms tends to zero, that is, $\Gamma_{n}^{n+1} \rightarrow 0$ as $n \rightarrow \infty$. Therefore, $\left\|u_{n+1}-u_{n}\right\|$ and $\left\|N\left(T u_{n}, A u_{n}\right)-N\left(T u^{*}, A u^{*}\right)\right\|$ converge to zero. Moreover, since the sequence $\left\{\Lambda\left(u_{n}, \rho_{n}\right)\right\}$ converges, it is bounded, and so is $\left\{u_{n}\right\}$ also according to (3.17).

Let $\bar{u}$ be a weak cluster point of the sequence $\left\{u_{n}\right\}$, and let $\left\{u_{n_{i}}\right\}$ be a subsequence converging weakly to $\bar{u}$. By using (3.13), since $K^{\prime}$ is Lipschitz continuous with constant $\kappa>0$ and $\rho_{n}>c_{1}$, it is known that for each $\nu \in D$,

$$
\begin{gathered}
\left\langle N\left(T u_{n}, A u_{n}\right)-w^{*}, \eta\left(v, u_{n+1}\right)\right\rangle+\varphi\left(v, u_{n+1}\right)-\varphi\left(u_{n+1}, u_{n+1}\right) \\
\geq-\left(\frac{1}{\rho_{n}}\right)\left\langle K^{\prime}\left(u_{n+1}\right)-K^{\prime}\left(u_{n}\right), \eta\left(v, u_{n+1}\right)\right\rangle \\
\geq-\left(\frac{\delta}{c_{1}}\right)\left\|K^{\prime}\left(u_{n+1}\right)-K^{\prime}\left(u_{n}\right)\right\|\left\|v-u_{n+1}\right\| .
\end{gathered}
$$

which hence implies that

$$
\begin{gathered}
\left\langle N\left(T u_{n_{i}}, A u_{n_{i}}\right)-w^{*}, \eta\left(v, u_{n_{i}+1}\right)\right\rangle+\varphi\left(v, u_{n_{i}+1}\right)-\varphi\left(u_{n_{i}+1}, u_{n_{i}+1}\right) \\
\geq-\left(\frac{\delta}{c_{1}}\right)\left\|K^{\prime}\left(u_{n_{i}+1}\right)-K^{\prime}\left(u_{n_{i}}\right)\right\|\left\|v-u_{n_{i}+1}\right\| .
\end{gathered}
$$

Since $\left\|u_{n+1}-u_{n}\right\| \rightarrow 0$, and $\left\{u_{n_{i}}\right\}$ converges weakly to $\bar{u}$, hence $\left\{u_{n_{i+1}}\right\}$ converges weakly to $\bar{u}$. Note that $K^{\prime}$ is sequentially continuous from the weak topology to the strong topology. Thus, it is easy to see that $\lim _{n \rightarrow \infty}\left\|K^{\prime}\left(u_{n_{i}+1}\right)-K^{\prime}\left(u_{n_{i}}\right)\right\|=0$. Since for each fixed $v \in D$, $u \rightarrow \eta(v, u)$ is sequentially continuous from the weak topology to the weak topology, and 
since the mapping $u \rightarrow N(T u, A u)$ is sequentially continuous from the weak topology to the strong topology, we infer that for each $v \in D$,

$$
\begin{aligned}
\left|\left\langle N\left(T u_{n_{i}}, A u_{n_{i}}\right)-w^{*}, \eta\left(v, u_{n_{i}+1}\right)\right\rangle-\left\langle N(T \bar{u}, A \bar{u})-w^{*}, \eta(v, \bar{u})\right\rangle\right| \\
\leq\left|\left\langle N\left(T u_{n_{i}}, A u_{n_{i}}\right)-N(T \bar{u}, A \bar{u}), \eta\left(v, u_{n_{i}+1}\right)\right\rangle\right| \\
\quad+\left|\left\langle N(T \bar{u}, A \bar{u})-w^{*}, \eta\left(v, u_{n_{i}+1}\right)-\eta(v, \bar{u})\right\rangle\right| \\
\leq|| N\left(T u_{n_{i}}, A u_{n_{i}}\right)-N(T \bar{u}, A \bar{u})||\left|\eta\left(v, u_{n_{i}+1}\right)\right| \mid \\
\quad+\left|\left\langle N(T \bar{u}, A \bar{u})-w^{*}, \eta\left(v, u_{n_{i}+1}\right)-\eta(v, \bar{u})\right\rangle\right| \longrightarrow 0 \quad \text { as } n \longrightarrow \infty .
\end{aligned}
$$

Consequently, letting $i \rightarrow \infty$ and using the weak continuity of $\varphi(\cdot, \cdot)$, we conclude from (3.29) that

$$
\left\langle N(T \bar{u}, A \bar{u})-w^{*}, \eta(v, \bar{u})\right\rangle+\varphi(v, \bar{u})-\varphi(\bar{u}, \bar{u}) \geq 0, \quad \forall v \in D .
$$

This shows that $\bar{u}$ is a solution of MQVLIP (1.3). Furthermore, $N(T \bar{u}, A \bar{u})=N\left(T u^{*}, A u^{*}\right)$ since $\lim _{n \rightarrow \infty}\left\|N\left(T u_{n_{i}}, A u_{n_{i}}\right)-N(T \bar{u}, A \bar{u})\right\|=0$ and $\lim _{n \rightarrow \infty} \| N\left(T u_{n}, A u_{n}\right)-N\left(T u^{*}\right.$, $\left.A u^{*}\right) \|=0$.

Finally, we claim that $\left\{u_{n}\right\}$ converges weakly to a solution of MQVLIP (1.3). Indeed, it suffices to show that $\left\{u_{n}\right\}$ has the unique weak cluster point. Let $\bar{u}$ and $\hat{u}$ be two weak cluster points of $\left\{u_{n}\right\}$. Then, both weak cluster points can be used as the above $u^{*}$ to define the Lyapunov function $\Lambda$. This yields two possible Lyapunov functions, denoted by $\bar{\Lambda}$ and $\hat{\Lambda}$, respectively. It was proven that $\Lambda\left(u_{n}, \rho_{n}\right)$ has a limit that may depend on the solution $u^{*}$ used to define $\Lambda$; then, the corresponding limits will be denoted by $\bar{l}$ and $\hat{l}$, respectively. Consider the subsequences $\left\{n_{i}\right\}$ and $\left\{m_{j}\right\}$ such that $\left\{u_{n_{i}}\right\}$ and $\left\{u_{m_{j}}\right\}$ converge weakly to $\bar{u}$ and $\hat{u}$, respectively. Then it is easy to see that $N(T \bar{u}, A \bar{u})=N(T \hat{u}, A \hat{u})$. Hence we get

$$
\begin{aligned}
\widehat{\Lambda}\left(u_{n_{i}}, \rho_{n_{i}}\right)= & K(\widehat{u})-K\left(u_{n_{i}}\right)-\left\langle K^{\prime}\left(u_{n_{i}}\right), \eta\left(\hat{u}, u_{n_{i}}\right)\right\rangle \\
& +\rho_{n_{i}}\left[\left\langle N(T \hat{u}, A \widehat{u})-w^{*}, \eta\left(u_{n_{i}}, \widehat{u}\right)\right\rangle+\varphi\left(u_{n_{i}}, \widehat{u}\right)-\varphi(\hat{u}, \widehat{u})\right] \\
= & K(\bar{u})-K\left(u_{n_{i}}\right)-\left\langle K^{\prime}\left(u_{n_{i}}\right), \eta\left(\bar{u}, u_{n_{i}}\right)\right\rangle \\
& +\rho_{n_{i}}\left[\left\langle N(T \bar{u}, A \bar{u})-w^{*}, \eta\left(u_{n_{i}}, \bar{u}\right)\right\rangle+\varphi\left(u_{n_{i}}, \bar{u}\right)-\varphi(\bar{u}, \bar{u})\right] \\
& +K(\widehat{u})-K(\bar{u})-\left\langle K^{\prime}\left(u_{n_{i}}\right), \eta(\hat{u}, \bar{u})\right\rangle \\
& +\rho_{n_{i}}\left[\left\langle N(T \hat{u}, A \hat{u})-w^{*}, \eta(\bar{u}, \hat{u})\right\rangle+\varphi\left(u_{n_{i}}, \hat{u}\right)-\varphi(\hat{u}, \hat{u})-\varphi\left(u_{n_{i}}, \bar{u}\right)+\varphi(\bar{u}, \bar{u})\right] \\
= & \bar{\Lambda}\left(u_{n_{i}}, \rho_{n_{i}}\right)+K(\widehat{u})-K(\bar{u})-\left\langle K^{\prime}\left(u_{n_{i}}\right), \eta(\hat{u}, \bar{u})\right\rangle \\
& +\rho_{n_{i}}\left[\left\langle N(T \hat{u}, A \hat{u})-w^{*}, \eta(\bar{u}, \hat{u})\right\rangle+\varphi\left(u_{n_{i}}, \hat{u}\right)-\varphi(\hat{u}, \hat{u})-\varphi\left(u_{n_{i}}, \bar{u}\right)+\varphi(\bar{u}, \bar{u})\right] .
\end{aligned}
$$


Now let $\rho_{*}=\lim _{n \rightarrow \infty} \rho_{n}$. Since $\hat{\Lambda}\left(u_{n_{i}}, \rho_{n_{i}}\right) \rightarrow \hat{l}$ and $\bar{\Lambda}\left(u_{n_{i}}, \rho_{n_{i}}\right) \rightarrow \bar{l}$, since $K^{\prime}$ is sequentially continuous from the weak topology to the strong topology, and since $\varphi(\cdot, \cdot)$ is weakly continuous, so, letting $n \rightarrow \infty$ and using the $\eta$-strong convexity of $K$, we conclude from (3.32) that

$$
\begin{aligned}
\hat{l}= & \bar{l}+K(\hat{u})-K(\bar{u})-\left\langle K^{\prime}(\bar{u}), \eta(\hat{u}, \bar{u})\right\rangle \\
& +\rho_{*}\left[\left\langle N(T \hat{u}, A \hat{u})-w^{*}, \eta(\bar{u}, \hat{u})\right\rangle+\varphi(\bar{u}, \hat{u})-\varphi(\hat{u}, \hat{u})\right] \\
\geq & \bar{l}+\left(\frac{\mu}{2}\right)\|\bar{u}-\hat{u}\|^{2} .
\end{aligned}
$$

By interchanging the role of $\bar{u}$ and $\hat{u}$ and of the subsequences $\left\{n_{i}\right\}$ and $\left\{m_{j}\right\}$, the same calculations yield

$$
\bar{l} \geq \hat{l}+\left(\frac{\mu}{2}\right)\|\hat{u}-\bar{u}\|^{2}
$$

Then, $0 \leq(\mu / 2)\|\bar{u}-\hat{u}\|^{2} \leq \hat{l}-\bar{l}$ and $0 \leq(\mu / 2) \| \hat{u}-\overline{\|}^{2} \leq \bar{l}-\hat{l}$. This implies that $\bar{u}=\hat{u}$.

Corollary 3.3. Let $D$ be a nonempty closed convex subset of a reflexive Banach space $B$ with the dual space $B^{*}$. Let $T, A: D \rightarrow B^{*}, N: B^{*} \times B^{*} \rightarrow B^{*}$, and $\eta: D \times D \rightarrow B$ be mappings. Let $w^{*} \in B^{*}$ and $\varphi: B \times B \rightarrow \mathbb{R} \bigcup\{+\infty\}$ be skew-symmetric and weakly continuous, such that for each $v \in B$, int $(\operatorname{dom} \varphi(\cdot, v)) \cap D \neq \varnothing$ and $\varphi(\cdot, v)$ is proper convex. Suppose that $K: D \rightarrow \mathbb{R}$ is $\eta$-strongly convex with constant $\mu>0$, and $K^{\prime}$ is sequentially continuous from the weak topology to the strong topology. Suppose also that

(i) the mapping $u \rightarrow N(T u, A u)$ is $\eta$-strongly monotone with constant $\beta>0$ and Lipschitz continuous with constant $L>0$;

(ii) $\eta$ is Lipschitz continuous with constant $\delta>0$, such that

(a) $\eta(u, v)=\eta(u, z)+\eta(z, v)$ for each $u, v, z \in D$,

(b) $\eta(\cdot, \cdot)$ is affine in the first variable,

(c) for each fixed $v \in D, u \rightarrow \eta(v, u)$ is sequentially continuous from the weak topology to the weak topology;

(iii) for each fixed $n \geq 0$ and $z \in D$,

$$
\left\{u \in D:\left\langle\rho_{n} N(T z, A z)-\rho_{n} w^{*}+K^{\prime}(u)-K^{\prime}(z), \eta(v, u)\right\rangle+\rho_{n} \varphi(v, u)-\rho_{n} \varphi(u, u) \geq 0\right\}
$$

is bounded for at least one $v \in D$.

Then, there is a unique solution $u_{n+1} \in D$ to problem (3.1) for each given iterate $u_{n}$. If

$$
c_{1}<\rho_{n}<\frac{2 \beta \mu}{\left(L^{2} \delta^{2}+c_{2}\right)}, \quad n \geq 0, \text { for some } c_{1}, c_{2}>0
$$


then $\left\{u_{n}\right\}$ converges strongly to a solution $u^{*} \in D$ of MQVLIP (1.3). Moreover, if $K^{\prime}$ is Lipschitz continuous with constant $\kappa>0$, then we have the posteriori error estimates:

$$
\left\|u_{n+1}-u^{*}\right\| \leq\left(\frac{\kappa \delta}{\beta \rho_{n}}+\frac{L \delta}{\beta}\right)\left\|u_{n+1}-u_{n}\right\| .
$$

Proof. We consider the variation of the function $\Phi$ in (3.15) for one stage of Algorithm 3.1,

$$
\Delta_{n}^{n+1}=\Phi\left(u_{n+1}\right)-\Phi\left(u_{n}\right)
$$

where $s_{1}=K\left(u_{n}\right)-K\left(u_{n+1}\right)-\left\langle K^{\prime}\left(u_{n}\right), \eta\left(u_{n}, u_{n+1}\right)\right\rangle$, and

$$
s_{2}=\left\langle K^{\prime}\left(u_{n}\right)-K^{\prime}\left(u_{n+1}\right), \eta\left(u^{*}, u_{n+1}\right)\right\rangle .
$$

By using $\eta$-strong convexity of $K$ and the fact that $\eta(u, v)=-\eta(v, u)$, for all $u, v \in D$, we have

$$
s_{1} \leq-\left(\frac{\mu}{2}\right)\left\|u_{n}-u_{n+1}\right\|^{2}
$$

Also, from (3.21) it follows that

$$
\begin{aligned}
s_{2} \leq & \rho_{n}\left[\left\langle N\left(T u_{n}, A u_{n}\right)-w^{*}, \eta\left(u^{*}, u_{n+1}\right)\right\rangle+\varphi\left(u^{*}, u_{n+1}\right)-\varphi\left(u_{n+1}, u_{n+1}\right)\right] \\
= & \rho_{n}\left\langle N\left(T u_{n+1}, A u_{n+1}\right)-N\left(T u^{*}, A u^{*}\right), \eta\left(u^{*}, u_{n+1}\right)\right\rangle \\
& +\rho_{n}\left\langle N\left(T u_{n}, A u_{n}\right)-N\left(T u_{n+1}, A u_{n+1}\right), \eta\left(u^{*}, u_{n+1}\right)\right\rangle \\
& +\rho_{n}\left[\left\langle N\left(T u^{*}, A u^{*}\right)-w^{*}, \eta\left(u^{*}, u_{n+1}\right)\right\rangle+\varphi\left(u^{*}, u_{n+1}\right)-\varphi\left(u_{n+1}, u_{n+1}\right)\right] .
\end{aligned}
$$

By using $\eta$-strong monotonicity of mapping $u \mapsto N(T u, A u)$, we have

$$
\rho_{n}\left\langle N\left(T u_{n+1}, A u_{n+1}\right)-N\left(T u^{*}, A u^{*}\right), \eta\left(u^{*}, u_{n+1}\right)\right\rangle \leq-\beta \rho_{n}\left\|u_{n+1}-u^{*}\right\|^{2} .
$$

By using Lipschitz continuity of mappings $u \mapsto N(T u, A u)$ and $\eta$, we get

$$
\begin{aligned}
\rho_{n}\langle N & \left.\left(T u_{n}, A u_{n}\right)-N\left(T u_{n+1}, A u_{n+1}\right), \eta\left(u^{*}, u_{n+1}\right)\right\rangle \\
& \leq \rho_{n} L \delta\left\|u_{n}-u_{n+1}\right\|\left\|u^{*}-u_{n+1}\right\| \\
& \leq\left(\frac{\mu}{2}\right)\left\|u_{n+1}-u_{n}\right\|^{2}+\left(\frac{\rho_{n}^{2} L^{2} \delta^{2}}{2 \mu}\right)\left\|u_{n+1}-u^{*}\right\|^{2} .
\end{aligned}
$$


By using skew-symmetry of $\varphi$ and the fact that $u^{*}$ is a solution of MQVLIP (1.3), we obtain

$$
\begin{aligned}
\rho_{n}\left[\left\langle N\left(T u^{*}, A u^{*}\right)-w^{*}, \eta\left(u^{*}, u_{n+1}\right)\right\rangle+\varphi\left(u^{*}, u_{n+1}\right)-\varphi\left(u_{n+1}, u_{n+1}\right)\right] \\
=-\rho_{n}\left[\left\langle N\left(T u^{*}, A u^{*}\right)-w^{*}, \eta\left(u_{n+1}, u^{*}\right)\right\rangle+\varphi\left(u_{n+1}, u^{*}\right)-\varphi\left(u^{*}, u^{*}\right)\right] \\
\quad-\rho_{n}\left[\varphi\left(u_{n+1}, u_{n+1}\right)-\varphi\left(u^{*}, u_{n+1}\right)-\varphi\left(u_{n+1}, u^{*}\right)+\varphi\left(u^{*}, u^{*}\right)\right] \\
\leq \rho_{n} \cdot 0+\rho_{n} \cdot 0=0 .
\end{aligned}
$$

Utilizing the estimates given in (3.42)-(3.44), we conclude from (3.41) that

$$
s_{2} \leq-\beta \rho_{n}\left\|u_{n+1}-u^{*}\right\|^{2}+\left(\frac{\mu}{2}\right)\left\|u_{n+1}-u_{n}\right\|^{2}+\left(\frac{\rho_{n}^{2} L^{2} \delta^{2}}{2 \mu}\right)\left\|u_{n+1}-u^{*}\right\|^{2} .
$$

This, together with (3.40), yields that

$$
\Delta_{n}^{n+1}=s_{1}+s_{2} \leq \rho_{n}^{2}\left(-\frac{\beta}{\rho_{n}}+\frac{L^{2} \delta^{2}}{2 \mu}\right)\left\|u_{n+1}-u^{*}\right\|^{2}
$$

Observe that (3.36) implies that

$$
\rho_{n}^{2}\left(-\frac{\beta}{\rho_{n}}+\frac{L^{2} \delta^{2}}{2 \mu}\right) \leq \rho_{n}^{2}\left(-\beta \frac{L^{2} \delta^{2}+c_{2}}{2 \beta \mu}+\frac{L^{2} \delta^{2}}{2 \mu}\right)=-\frac{\rho_{n}^{2} c_{2}}{2 \mu} \leq-\frac{c_{1}^{2} c_{2}}{2 \mu} .
$$

Now substituting the estimate (3.47) in (3.46), we derive

$$
\Delta_{n}^{n+1} \leq\left(-\frac{c_{1}^{2} c_{2}}{2 \mu}\right)\left\|u_{n+1}-u^{*}\right\|^{2}
$$

This shows that $\Delta_{n}^{n+1}$ is negative unless $u_{n+1}=u^{*}$. The sequence $\left\{\Phi\left(u_{n}\right)\right\}$ is strictly decreasing. But since it is positive, it must converge and the difference between two consecutive terms tends to zero, that is, $\Delta_{n}^{n+1} \rightarrow 0$ as $n \rightarrow \infty$. Therefore, it follows from (3.48) that $\left\{u_{n}\right\}$ converges strongly to $u^{*}$.

Now, by using (3.13) with $v=u^{*}$, we infer that

$$
\begin{aligned}
& \left\langle K^{\prime}\left(u_{n+1}\right)-K^{\prime}\left(u_{n}\right), \eta\left(u^{*}, u_{n+1}\right)\right\rangle+\rho_{n}\left\langle N\left(T u_{n}, A u_{n}\right)-w^{*}, \eta\left(u^{*}, u_{n+1}\right)\right\rangle \\
& \quad+\rho_{n} \varphi\left(u^{*}, u_{n+1}\right)-\rho_{n} \varphi\left(u_{n+1}, u_{n+1}\right) \geq 0 .
\end{aligned}
$$

By using Lipschitz continuity of mappings $K^{\prime}, \eta$, and $u \mapsto N(T u, A u)$, skew-symmetry of $\varphi, \eta$-strong monotonicity of $u \rightarrow N(T u, A u)$, and the fact that $u^{*}$ is a solution of MQVLIP 
(1.3), we have that

$$
\begin{aligned}
\kappa \delta \| u_{n+1} & -u_{n}\|\| u_{n+1}-u^{*}\left\|+\rho_{n} L \delta\right\| u_{n+1}-u_{n}\|\| u_{n+1}-u^{*} \| \\
\geq & \left\langle K^{\prime}\left(u_{n+1}\right)-K^{\prime}\left(u_{n}\right), \eta\left(u^{*}, u_{n+1}\right)\right\rangle \\
& +\rho_{n}\left\langle N\left(T u_{n}, A u_{n}\right)-N\left(T u_{n+1}, A u_{n+1}\right), \eta\left(u^{*}, u_{n+1}\right)\right\rangle \\
\geq & \rho_{n}\left\langle N\left(T u_{n+1}, A u_{n+1}\right)-N\left(T u^{*}, A u^{*}\right), \eta\left(u_{n+1}, u^{*}\right)\right\rangle \\
& +\rho_{n}\left[\left\langle N\left(T u^{*}, A u^{*}\right)-w^{*}, \eta\left(u_{n+1}, u^{*}\right)\right\rangle+\varphi\left(u_{n+1}, u^{*}\right)-\varphi\left(u^{*}, u^{*}\right)\right] \\
& +\rho_{n}\left[\varphi\left(u_{n+1}, u_{n+1}\right)-\varphi\left(u^{*}, u_{n+1}\right)-\varphi\left(u_{n+1}, u^{*}\right)+\varphi\left(u^{*}, u^{*}\right)\right] \\
\geq & \rho_{n}\left\langle N\left(T u_{n+1}, A u_{n+1}\right)-N\left(T u^{*}, A u^{*}\right), \eta\left(u_{n+1}, u^{*}\right)\right\rangle \\
\geq & \beta \rho_{n}\left\|u_{n+1}-u^{*}\right\|^{2},
\end{aligned}
$$

which hence implies that

$$
\kappa \delta|| u_{n+1}-u_{n}||\left\|u_{n+1}-u^{*}||+\rho_{n} L \delta\right\| u_{n+1}-u_{n}\|\| u_{n+1}-u^{*}\left\|\geq \beta \rho_{n}\right\| u_{n+1}-u^{*} \|^{2} .
$$

We obtain inequality (3.37) after division by $\left\|u_{n+1}-u^{*}\right\|$, which we assume nonzero; otherwise, the result is trivial.

\section{Acknowledgments}

The first author was partially supported by the Teaching and Research Award Fund for Outstanding Young Teachers in Higher-Education Institutions of MOE, China, and the Dawn Program Foundation in Shanghai. The second author is grateful to the Department of Mathematical Sciences, King Fahd University of Petroleum \& Minerals, Dhahran, Saudi Arabia, for providing excellent research facilities. The third author was partially supported by a grant from the National Science Council.

\section{References}

[1] Q. H. Ansari and J.-C. Yao, Iterative schemes for solving mixed variational-like inequalities, Journal of Optimization Theory and Applications 108 (2001), no. 3, 527-541.

[2] A. S. Antipin, Iterative gradient prediction-type methods for computing fixed points of extremal mapping, Parametric Optimization and Related Topics, IV (Enschede, 1995) (J. Guddat, H. Th. Jonden, F. Nizicka, G. Still, and F. Twitt, eds.), Approx. Optim., vol. 9, Lang, Frankfurt am Main, 1997, pp. 11-24.

[3] N. H. Dien, Some remarks on variational-like and quasivariational-like inequalities, Bulletin of the Australian Mathematical Society 46 (1992), no. 2, 335-342.

[4] X. P. Ding and J.-C. Yao, Existence and algorithm of solutions for mixed quasi-variational-like inclusions in Banach spaces, Computers \& Mathematics with Applications 49 (2005), no. 5-6, 857-869. 
[5] K. Fan, A generalization of Tychonoff's fixed point theorem, Mathematische Annalen 142 (1961), no. 3, 305-310.

[6] R. Glowinski, J.-L. Lions, and R. Trémolières, Numerical Analysis of Variational Inequalities, Studies in Mathematics and Its Applications, vol. 8, North-Holland, Amsterdam, 1981.

[7] M. A. Hanson, On sufficiency of the Kuhn-Tucker conditions, Journal of Mathematical Analysis and Applications 80 (1981), no. 2, 545-550.

[8] N.-J. Huang and C.-X. Deng, Auxiliary principle and iterative algorithms for generalized set-valued strongly nonlinear mixed variational-like inequalities, Journal of Mathematical Analysis and Applications 256 (2001), no. 2, 345-359.

[9] S. Karamardian, The nonlinear complementarity problem with applications. II, Journal of Optimization Theory and Applications 4 (1969), no. 3, 167-181.

[10] C.-H. Lee, Q. H. Ansari, and J.-C. Yao, A perturbed algorithm for strongly nonlinear variationallike inclusions, Bulletin of the Australian Mathematical Society 62 (2000), no. 3, 417-426.

[11] M. A. Noor, Variational-like inequalities, Optimization 30 (1994), no. 4, 323-330.

[12] A. H. Siddiqi, Q. H. Ansari, and R. Ahmad, Some remarks on variational-like inequalities, Mathematics and Its Applications in Industry and Business (A. H. Siddiqi and K. Ahmad, eds.), Narosa, New Delhi, 2000, pp. 101-108.

[13] P. Tseng, Further applications of a splitting algorithm to decomposition in variational inequalities and convex programming, Mathematical Programming, Series B 48 (1990), no. 2, 249-263.

[14] L.-C. Zeng, Iterative algorithm for finding approximate solutions of a class of mixed variational-like inequalities, Acta Mathematicae Applicatae Sinica. English Series 20 (2004), no. 3, 477-486.

[15] L. C. Zeng, Iterative approximation of solutions to generalized set-valued strongly nonlinear mixed variational-like inequalities, Acta Mathematica Sinica (Chinese Series) 48 (2005), no. 5, 879888.

[16] L. C. Zeng, S. Schaible, and J.-C. Yao, Iterative algorithm for generalized set-valued strongly nonlinear mixed variational-like inequalities, Journal of Optimization Theory and Applications $\mathbf{1 2 4}$ (2005), no. 3, 725-738.

[17] D. L. Zhu and P. Marcotte, Co-coercivity and its role in the convergence of iterative schemes for solving variational inequalities, SIAM Journal on Optimization 6 (1996), no. 3, 714-726.

Lu-Chuan Ceng: Department of Mathematics, Shanghai Normal University, Shanghai 200234, China E-mail address: zenglc@shnu.edu.cn

Qamrul Hasan Ansari: Department of Mathematics, Aligarh Muslim University,

Aligarh 202002, India

Current address: Department of Mathematical Sciences, King Fahd University of Petroleum \&

Minerals, P.O. Box 1169, Dhahran 31261, KSA

E-mail address: qhansari@kfupm.edu.sa

Jen-Chih Yao: Department of Applied Mathematics, National Sun Yat-sen University,

Kaohsiung 804, Taiwan

E-mail address: yaojc@math.nsysu.edu.tw 